Ann. Biol. anim. Bioch. Biophys., 1977, 17 (4), 597-602.

\title{
Influence de la composition du régime alimentaire sur le transit digestif et l'absorption intestinale des acides aminés chez le poulet
}

\author{
par M. LARBIER, N. CARBO BAPTISTA, J. C. BLUM
}

Station de Recherches Avicoles, I. N.R. A. Nouzilly 37380 Monnaie.

Summary. Effect of diet composition on digestive transit and amino acid intestinal absorption in chicken.

Diets were offered ad libitum to 3 week-old chickens, using a factorial scheme with 2 energy levels ( 3.1 and $2.7 \mathrm{kcal}$ of metabolisable energy per $\mathrm{g}), 2$ protein levels $(21.1$ and 16 p. 100) and 2 lysine levels (1.18 and 0.6 p. 100).

Results showed that digestive transit rate depended only on energy supply, and decreased with a lipid-rich diet. The lysine absorption rate depended on dietary supply of nitrogenous matter, lysine and energy. With a high energy level, it increased in case of protein deficiency; this was accentuated when there was lysine deficiency. Energy level being essential, lysine absorption rate always decreased when this level was reduced.

These observations do not apply to all parts of the small intestine in the same way; they were more marked in the duodenum and ileum than in the jejunum.

\section{Introduction.}

Chez les mammifères, l'influence du régime alimentaire sur la vitesse d'absorption des acides aminés fait l'objet de nombreux travaux depuis que Neame ef Wiseman (1959) puis Kershaw, Neame et Wiseman (1960) ont montré que la restriction énergétique entraîne chez le rat une augmentation du transport actif de l'histidine.

Chez le poulet, l'adaptation de la physiologie de l'intestin grêle aux conditions nutritionnelles n'a fait l'objet que de rares études essentiellement centrées sur l'effet des vitamines et revues récemment par Kan (1975). En ce qui concerne l'apport alimentaire de protéines ou de nutriments énergétiques, seule l'influence du taux protidique sur la vitesse d'absorption de quelques acides aminés neutres (glycine, alanine, leucine et méthionine) a été envisagée par Sheerin et Bird (1972).

Dans notre essai, nous avons fait varier les teneurs du régime en constituants énergétiques, en matières azotées totales et en lysine, et comparé l'influence de ces variations sur le transit digestif, la vitesse d'absorption de la lysine dans l'intestin grêle et les performances de croissance du poulet de chair. 


\section{Małériel ef méthodes.}

Les poulets de type chair sont élevés en cages individuelles sous $22 \mathrm{~h}$ de lumière par nycthémère. Ils disposent tous d'un même régime complet jusqu'à l'âge de trois semaines. Ils sont alors pesés et répartis en huit lots homogènes de dix animaux. Chaque lot reçoit l'un des huit régimes expérimentaux dont la composition figure au tableau 1. Ces régimes sont constitués selon un plan factoriel comportant deux taux énergétiques ( 3100 et $2700 \mathrm{kcal}$ d'énergie métabolisable par $\mathrm{kg}$ ), deux teneurs différentes en protéines totales $(21$ et 16 p. 100) ef deux concentrations distinctes en lysine $(1,2$ ef 0,6 p. 100$)$.

\section{TABLEAU 1}

Composition et caractéristiques des régimes alimentaires

\begin{tabular}{|c|c|c|c|c|c|c|c|c|}
\hline Régime & $\mathbf{T}$ & $E$ & $P$ & $\mathrm{~L}$ & LP & LE & PE & LPE \\
\hline \multicolumn{9}{|l|}{ Composition } \\
\hline 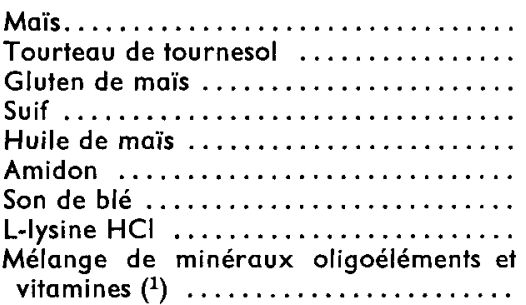 & $\begin{array}{l}50 \\
30 \\
6 \\
4,5 \\
0,5 \\
4 \\
\overline{0,53} \\
4,6\end{array}$ & $\begin{array}{l}50 \\
30 \\
6 \\
-0,5 \\
5 \\
3,5 \\
0,53 \\
4,6\end{array}$ & $\begin{array}{l}62 \\
22 \\
2 \\
3 \\
0,5 \\
4,5 \\
0,50 \\
0,90 \\
4,6\end{array}$ & $\begin{array}{l}50 \\
30 \\
6 \\
4,5 \\
0,5 \\
4 \\
0,50 \\
- \\
4,6\end{array}$ & $\begin{array}{l}62 \\
22 \\
2 \\
3 \\
0,5 \\
4,5 \\
1,25 \\
0,15 \\
4,6\end{array}$ & $\begin{array}{l}50 \\
30 \\
6 \\
- \\
0,5 \\
5 \\
4 \\
- \\
4,6\end{array}$ & $\begin{array}{l}62 \\
22 \\
2 \\
- \\
0,5 \\
4 \\
4,0 \\
0,90 \\
4,6\end{array}$ & $\begin{array}{l}62 \\
22 \\
2 \\
-0,5 \\
4 \\
4,75 \\
0,15 \\
4,6\end{array}$ \\
\hline
\end{tabular}

\section{Caractéristiques}

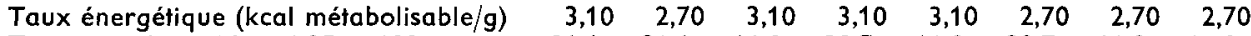

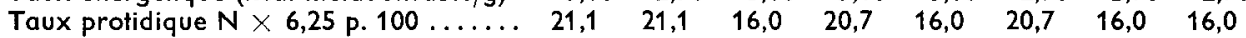

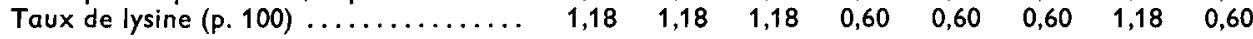

(1) Apportant pour $100 \mathrm{~kg}$ d'aliment : phosphate bicalcique 1,75 kg ; carbonate de Ca 1,50 kg ; sel vétérinaire $0,5 \mathrm{~kg}$; oligoéléments $\mathrm{UCAAB} 0,20 \mathrm{~kg}$; vitamine A $2200000 \mathrm{Ul}$; vitamine D3 $280000 \mathrm{Ul}$; vitamine $E 7,5 \mathrm{~g}$; nicotinamide $1,4 \mathrm{~g}$; panthothenate de $\mathrm{Ca} 800 \mathrm{mg}$; vitamine $\mathrm{B} 12$ 0,14 mg; vifamine $\mathrm{B} 2600 \mathrm{mg}$; choline $60 \mathrm{~g}$; BHT $18 \mathrm{~g}$.

A l'âge de cinq semaines, les animaux mis à jeôn depuis $18 \mathrm{~h}$ sont pesés. Sur 5 poulets de chaque lot maintenus sous anesthésie à l'éther pendant la durée de la manipulation, on isole trois anses intestinales, l'une comprenant l'ensemble du duodénum, les deux autres de $15 \mathrm{~cm}$ chacune sont situées de part ef d'autre du diverticule de Meckel. Les anses ainsi constituées sont perfusées simultanément in situ et en circuit ouvert (débit $0,42 \mathrm{ml} / \mathrm{mn}$ ) pendant deux heures avec une solution de Krebs-Ringer phosphate $(\mathrm{pH}=7,4)$ chauffée à $39^{\circ} \mathrm{C}$ et renfermant de la L-lysine, $\mathrm{HCl}(7 \mathrm{mM} / \mathrm{l})$.

La lysine retenue au cours de la perfusion mesure l'absorption. Elle est déter- 
minée par différence, la fraction qui demeure dans le perfusat étant dosée après chromatographie sur colonne de résine échangeuse d'ions selon la méthode de Moore, Spackman et Stein (1958) à l'aide d'un autoanalyseur Technicon.

Pour estimer la vitesse du transit digestif, les 5 poulets restant de chaque lot reçoivent pendant trois heures une ration alimentaire de même composition que celle des jours précédents, mais additionnée de 3 p. 100 d'oxyde de chrome.

Les excreta récoltés pendant les quatre heures qui suivent la fin du repas sont calcinés. L'oxyde de chrome contenu dans les cendres est dosé par spectrophotométrie selon la méthode de Mathieson (1970) à l'aide d'un autoanalyseur Technicon.

\section{Résultats.}

Les performances zootechniques réalisées pendant les deux semaines d'expérimentation vérifient a posteriori le déséquilibre par défaut d'énergie, de protéines totales ou de lysine dans les régimes renfermant respectivement $2700 \mathrm{kcal}$ d'énergie métabolisable, 16 p. 100 de protéines et 0,6 p. 100 de lysine (tabl. 2).

\section{TABLEAU 2}

Influence de la composition du régime alimentaire sur les performances des poulets entre 3 et 5 semaines d'âge

\begin{tabular}{|c|c|c|c|c|c|c|c|c|}
\hline Régimes & $T$ & E & LE & $\mathrm{PE}$ & LP & $\mathbf{P}$ & $\mathbf{L}$ & LPE \\
\hline Poids vif initial (G). & 394 & 391 & 392 & 389 & 393 & 387 & 390 & 391 \\
\hline Gain de Poids (G) $\left(^{*}\right)$ & $\begin{array}{c}413^{A} \\
\pm 9\end{array}$ & $\begin{array}{r}336 \mathrm{~B} \\
\pm 16\end{array}$ & $\begin{array}{r}359 \mathrm{~B} \\
\pm 33\end{array}$ & $\begin{array}{r}261^{\mathrm{C}} \\
\pm 23\end{array}$ & $\begin{array}{r}374^{\mathrm{B}} \\
\pm 16\end{array}$ & $\begin{array}{l}200 \mathrm{C} \\
\pm 18\end{array}$ & $\begin{array}{r}381 \mathrm{~B} \\
\pm 14\end{array}$ & $\begin{array}{l}295^{C} \\
\pm 6\end{array}$ \\
\hline dice de consommation & 1,96 & 2,23 & 2,53 & 3,21 & 2,43 & 3,43 & 2,68 & 2,6 \\
\hline
\end{tabular}

$\left({ }^{*}\right)$ Les valeurs suivies de lettres différentes sont significativement différentes $(P \leqslant 0,01)$.

Tous ces déséquilibres entraînent une diminution du gain de poids et une augmentation de l'indice de consommation. II faut cependant noter que l'effet dépressif de la déficience partielle en lysine sur le gain de poids est particulièrement marqué. Il est encore plus accentué lorsque le régime alimentaire est à la fois déficient en lysine et en énergie (régime LE).

Il en est de même pour l'indice de consommation. Les valeurs les plus élevées sont enregistrées avec les régimes déficients soit en lysine et en énergie soit seulement en lysine.

Les quantités d'oxyde de chrome retrouvées dans les fèces révèlent une importante variabilité individuelle du transit digestif (tabl. 3). On peut, cependant, noter un effet exclusif et significatif du taux énergétique. Les animaux ayant reçu une ration renfermant $2700 \mathrm{kcal} / \mathrm{kg}$ d'énergie métabolisable, ont excrété pendant les quatre 
heures qui suivent le repas, entre 53,7 et 63,3 p. 100 de l'oxyde de chrome ingéré. En revanche, lorsque le régime alimentaire contient $3100 \mathrm{kcal} / \mathrm{kg}$, les valeurs obtenues oscillent entre 40 et 45 p. 100.

TABLEAU 3

Influence de la composition du régime alimentaire sur la vifesse d'absorption de la lysine mesurée in vivo en $\mu \mathrm{M} / 15 \mathrm{~cm} / 30 \mathrm{~min}$ et le transit digestif ( $p .100 \mathrm{de} \mathrm{Cr}_{2} \mathrm{O}_{3}$ excrété $4 \mathrm{~h}$ après le repas)

\begin{tabular}{|c|c|c|c|c|}
\hline Régimes & Duodénum (1) & Jéjunum (1) & lléon ( $\left.{ }^{1}\right)$ & $\begin{array}{l}\text { P. } 100 \mathrm{Cr}_{2} \mathrm{O}_{3} \\
\text { dans les fèces }\left({ }^{2}\right)\end{array}$ \\
\hline 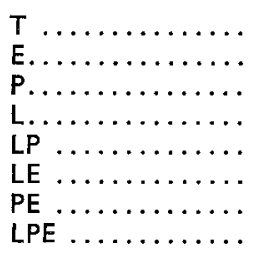 & $\begin{array}{r}17,2^{\mathrm{a}} \pm 2,2 \\
9,6^{\mathrm{b}} \pm 0,7 \\
26,8^{\mathrm{c}} \pm 2,5 \\
32,3^{\mathrm{c}} \pm 2,8 \\
32,5^{\mathrm{c}} \pm 2,8 \\
9,4^{\mathrm{b}} \pm 0,9 \\
12,7^{\mathrm{b}} \pm 1,1 \\
9,1^{\mathrm{b}} \pm 1,9\end{array}$ & $\begin{array}{l}21,9^{\mathrm{a}} \pm 1,7 \\
17,6^{\mathrm{b}} \pm 0,8 \\
19,9^{\mathrm{a}} \pm 1,2 \\
20,7^{\mathrm{a}} \pm 1,6 \\
18,2^{\mathrm{a}} \pm 1,2 \\
16,0^{\mathrm{b}} \pm 1,4 \\
16,7^{\mathrm{b}} \pm 2,0 \\
19,2^{\mathrm{a}} \pm 1,9\end{array}$ & $\begin{array}{l}25,9^{\mathrm{a}} \pm 1,7 \\
22,3^{\mathrm{b}} \pm 1,1 \\
31,3^{\mathrm{c}} \pm 2,0 \\
32,0^{\mathrm{c}} \pm 2,1 \\
34,3^{\mathrm{c}} \pm 1,4 \\
19,6^{\mathrm{b}} \pm 2,0 \\
25,1^{\mathrm{a}} \pm 3,2 \\
23,2^{\mathrm{a}} \pm 2,1\end{array}$ & $\begin{array}{l}45,0^{\mathrm{a}} \pm 1,5 \\
63,3^{\mathrm{b}} \pm 3,3 \\
49,0^{\mathrm{a}} \pm 1,9 \\
43,7^{\mathrm{a}} \pm 1,9 \\
40,0^{\mathrm{a}} \pm 4,6 \\
62,0^{\mathrm{b}} \pm 4,6 \\
53,7^{\mathrm{b}} \pm 3,2 \\
55,7^{\mathrm{b}} \pm 2,8\end{array}$ \\
\hline
\end{tabular}

(1) Pour un segment intestinal donné, les valeurs suivies de lettres différentes sont très significativement différentes $(P \leqslant 0,01)$.

(2) Les valeurs suivies de lettres différentes sont significativement différentes $(P \leqslant 0,05)$.

Les résultats relatifs à la vitesse d'absorption de la lysine peuvent être analysés d'une part en comparant les trois segments de l'intestin grêle, d'autre part en recherchant l'influence de la composition du régime alimentaire (tabl. 3). Chez les animaux nourris avec un aliment équilibré (régime T), la vitesse d'absorption de la lysine mesurée in vivo est plus élevée dans l'iléon que dans le jéjunum ; ce dernier absorbant lui-même moins rapidement la lysine que le duodénum. Cette hiérarchie entre les trois segments est parfois modifiée lorsque varient les apports alimentaires d'énergie, protéines ou lysine.

D'une façon générale, l'amplitude des variations de la vitesse d'absorption de la lysine est plus grande pour le duodénum que pour les deux autres segments.

La subdéficience en énergie a un effet inverse de celui d'un apport insuffisant en protéines totales ou seulement en lysine. Dans le premier cas, on enregistre une diminution significative dans l'ensemble de l'intestin grêle, particulièrement au niveau du duodénum. En revanche la subdéficience en lysine ou en protéines totales entraîne une augmentation dans les parties proximale et distale de l'intestin grêle, les valeurs obtenues pour le jéjunum n'étant pas modifiées.

Chez les animaux nourris avec des régimes déséquilibrés à la fois en énergie et protéines, énergie et lysine, lysine ef protéines ou en énergie, protéines et lysine, les résultats obtenus sont de même nature que si le régime comportait une simple déficience. Il faut cependant noter l'effet prépondérant de l'apport alimentaire en énergie : la vitesse d'absorption de la lysine diminue dans tous les cas où le taux énergétique de l'aliment est réduit. 


\section{Discussion.}

La réduction des apports alimentaires en énergie, en protéines et en lysine conduit à des baisses de performances qui étaient prévisibles compte tenu des besoins du poulet de chair (Journées Volailles de Consommation, 1975). En revanche, l'influence du taux énergétique sur le transit digestif mérite d'être commentée. Les études réalisées chez les oiseaux sont plutôt rares. II a été montré que la vitesse du transit variait avec les caractéristiques de l'aliment; liquides ou solides, granulés ou farines ne sont pas évacués selon la même cinétique (Jacquot, Le Bars et Simmonet, 1958 ; Hill, 1971). Tuckey, March et Biely (1958) ont distribué des régimes de composition différente en particulier par leur teneur en graisses. Ils ont constaté que le temps nécessaire à l'apparition du marqueur (oxyde ferrique) dans les fientes était invariable. En fait ce temps qui est très court $(2 \mathrm{~h} 30)$ ne mesure pas le transit. Pour avoir collecté les fientes à divers moments après le repas, nous savons que le marqueur n'est pas éliminé à une vitesse constante (résultats non publiés). Le temps choisi dans notre essai correspond sensiblement à la valeur admise comme durée moyenne du transit chez le poulet en croissance (Hill, 1971). Les régimes à taux énergétique élevé (3100 kcal) renferment un supplément de graisse (3 à 4,5 p. 100 de suif) qui peut être rendu responsable du ralentissement de l'évacuation digestive. L'action inhibitrice des graisses sur la vidange gastrique est bien comme chez les mammifères (Laplace, 1975). Elle concerne probablement les oiseaux, se traduisant ainsi que le montre notre essai par un transit global plus lent.

L'influence du régime alimentaire sur la vitesse d'absorption des acides aminés a été essentiellement étudiée chez des animaux soumis à des restrictions nutritionnelles très sévères : jeûne intermittent (Kershaw, Neame et Wieseman 1960), régime totalement déficient en nutriments énergétiques ou protidiques (Adibi et Allen, 1970) ou en un acide aminé (Shishova, 1963). L'effet du jeûne attribué en premier lieu à une carence en énergie, se traduit par une diminution de la vitesse d'absorption des acides aminés (Newey, Sanford et Smyth, 1970). A l'inverse, lors d'une déficience en protéines totales (Shimomura, 1964) ou en un acide aminé essentiel (Shishova, 1963), l'absorption est stimulée. Les résultats obtenus dans notre essai, dans le cas de la lysine confirment ces tendances bien que les régimes alimentaires ditribués soient nettement moins déséquilibrés que ceux utilisés par les différents auteurs que nous avons cités. Nos observations s'opposent cependant, en partie, à celles de Sheerin et Bird (1972) : distribuant un régime déficient en protéines à des poulets âgés de 7 à 8 semaines, ces auteurs constatent que l'absorption des acides aminés neutres n'est pas modifiée. Il faut donc admettre une réaction différente liée à l'âge (nos poulets étaient plus jeunes) ou à la nature des acides aminés, le processus d'adaptation observé avec la lysine n'ayant pas forcément un caractère général.

En définitive, on peut affirmer que la composition du régime influence la physiologie digestive et tout particulièrement la vitesse d'absorption des acides aminés, chez le poulet en croissance. Les facteurs nutritionnels qui assurent l'épargne des protéines influencent les activités enzymatiques de dégradation (Nakano et Ashida, 1970). Nous montrons qu'ils peuvent aussi modifier le transport à travers les membranes. Cela est vrai pour la lysine, qui occupe peut-être une place privilégiée. On 
sait en effet que le catabolisme de cet acide aminé est particulièrement bien contrôlé (Chu et Hegsted, 1976). On peut se demander s'il en est de même pour l'absorption digestive. L'usage d'autres acides aminés nous permettra sans doute de répondre ultérieurement à cette question.

Commission CNERNA Digestion-Absorption, Tours, 13 novembre 1976.

\section{Références}

ADIBI S. A., ALLEN E. R., 1970. Impaired jejunal absorption rates of essential amino acids induced by either dietary caloric or protein deprivation in man. Gastroenterology, 59, 404.

CHU S. H.W., HEGSTED D. M., 1976. Adaptative response of lysine and threonine degrading enzymes in adult rats. J. Nutr., 106, 1089-1096.

HILL K. J., 1971. The physiology of digestion in Physiology and biochemistry of the domestic fowl. vol. 1, 25-49. Bell and Freeman édit.

JACQUOT R., LE BARS H., SIMONNET H., 1958. Nutrition animale. Vol. I. Données générales sur la nutrition ef l'alimentation. J. B. Baillère et Fils, Paris.

Journées Volailles de consommation, 16 ef 17 ocfobre 1975, Nouzilly, I. N. R. A., 1 vol., 194 pp.

KAN C. A., 1975. The intestinal absorption of amino acids and peptides with special reference to the domestic fowl: A litterature review. WId's Poult. Sci. J., 31, 46-56.

KERSHAW T. G., NEAME K. D., WISEMAN G., 1960. The effect of semistarvation on absorption by the rat small intestine in vitro and in vivo. J. Physiol., 152, 182.

LAPLACE J. P., 1975. Le transit digestif chez les monogastriques II. Phénomènes moteurs et mouvements des digesta. Ann. Zoolech., 24, 489-552.

MATHIESON J., 1970. The automated estimation of chromic oxide. Proc. Nutr. Soc. 29, 30A-31A.

MOORE S., SPACKMAN D. H., STEIN W. H., 1958. Chromatography of amino acids on sulfonated polystyrene resins. Analyt. Chem., 30, 1185-1190.

NAKANO K., ASHIDA K., 1970. Effect of dietary carbohydrate and fat on amino acid degrading enzymes in relation to their protein sparing action. J. Nutr., 100, 208-216.

NEAME K. D., WISEMAN G., 1959. The effect of dief on intestinal active transport. J. Physiol., 146, $10 \mathrm{P}$.

NEWEY H., SANFORD P. A., SMYTH D. H., 1970. Effects of fasting on intestinal transfer of sugars and amino acids in vitro. J. Physiol., 208, 705.

SHEERIN H. E., BIRD F. H., 1972. Studies on the competitive absorption of neutral L-amino acids from the avian small intestine. J. Nutr., 102, 1563-1568.

SHIMOMURA A., 1964. Adaptative increase of intestinal transport of L-amino acid in rats fed on proteins of inferior quality. Osaka Univ. Med. J., 16, 415.

SHISHOVA O. A., 1963. Effect of alimentary factor on absorption of amino acid mixtures in intestines. Vop. Piton, 22, 3.

TUCKEY R., MARCH B. E., BIELY J., 1958. Diet and the rate of food passage in the growing chick. Poult. Sci., 37, 786-792. 